\title{
AC 2010-1226: MAXIMISING THE IMPACT OF CREATIVE AND INNOVATIVE ACTIVITIES WITHIN THE CONSTRAINTS OF DEFINED EDUCATION STRUCTURES
}

Niall Seery, University of Limerick

Donal Canty, University of Limerick

Ronan Dunbar, University of Limerick 


\title{
Maximising the impact of creative and innovative activities within the constraints of defined education structures
}

\begin{abstract}
In recent years Ireland has committed to significant educational reform in technological education. With the successful implementation of two new subjects at Senior Cycle (pre third level) that are predicated on creativity and innovation, design is now an essential facet of the second level curriculum. This new focus encourages students to become enterprising, creative and empowered during their learning experience.

This paper centres on defining an innovative pedagogical framework to facilitate the fluidity, dynamics and personalisation of educational interventions within an initial technology teacher education programme. Focusing on the teacher as the primary agents for change, this study explores the effect of forming the norms and practices of pre-service teachers in effectively achieving the desired outcomes of the new curriculum at second level.

The approach taken, explores the novel use of personal assessable technologies that enable the collection, synthesis, and narrative capabilities essential to the effective stimulation, development and management of inspiration. This study argues the value of non-criterion referenced activities; in engaging student teachers in a constructive critique of education and facilitates the derivation of their own value system. The findings discuss the demands and benefits of creative and innovative technological strategies within the constraints of a traditional education model. As a result the paper recommends a blueprint for engaging students in design and creative activities within technology and presents these benefits in terms of holistic student development.
\end{abstract}




\section{Technology Education” within the Irish Educational Framework}

The Irish Education System is a three-tiered system that begins at Primary Level and continues to Second Level. The second level system is divided into two distinct programs, the Junior and Senior Cycle. The Leaving Certificate is completed at the end of the Senior Cycle and it is through the pupils' level of achievement in this examination procedure that enables them to enter courses of study at third level.

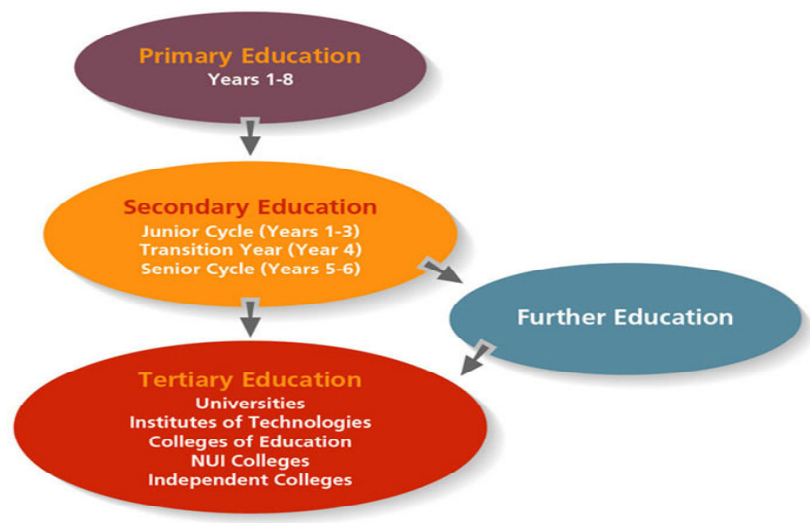

Figure 1 - Structure of Irish Education

Within the Junior Cycle (the initial three year program) of the second level system pupils are for the first time provided with options of studying technology education through the provision of four syllabi. These subjects are Metalwork, Woodwork, Technical Graphics and Technology and their main aims are to contribute to and develop the skills and knowledge required to take further course of study in their later years of education. The Senior Cycle (the final two years of the Second Level system) offers Technology Education through four syllabi, thereby giving progression from technology education in the Junior Cycle. The subjects are Engineering, Construction Studies, Design and Communication Graphics and Technology.

The Third Level system in Ireland then offers a number of different areas of study within the area of Technology Education to successful school leavers as would be expected of all national Tertiary Level education systems. This paper is concerned with and refers to both the second level and third level education systems as the cohort that is being analysed throughout the methodology are undertaking a concurrent teacher education program at the University of Limerick to equip them to teach the technology subjects at second level.

\section{Fostering "New Skills" in Technology Education}

Technology Education is recognised as a fundamental discipline within the Irish Education System. The strength of Technology Education and the quality of the students graduating from courses of study in the third level institutes in the country is often seen as one of the main reasons for Ireland's previous economic success. The recent implementation of two new syllabi at Senior Cycle (pre third level) shows how the importance of the subject area is still valued. Design and Communication Graphics and Technology are the two subjects that were recently formed and successfully implemented to respond to the changing needs of the $21^{\text {st }}$ century's global society. In the preface statements of these syllabi it is clearly outlined that the intentions of their implementation is to incorporate the principles of design and realisation in a creative manner ${ }^{1}$.

It is clear that our education system is no longer concerned with the type of vocational training approach that traditionally predominated technology education in this country. 
The critical aims of these new syllabi display how the focus of technology education has changed, and now focusing on endorsing a cohort of students to be equipped with skills of design and realisation and the ability to apply these skills by thinking and acting imaginatively and creatively. Through the technology subject area it is recognised that students are presented with a learning environment that is not as rigid as other areas of the curriculum. Academic subjects such as maths and science can promote convergent thinking that usually leads to one solution to a predefined problem, whereas technology subjects orientate around divergent thinking where the student is presented with a problem and can apply their own personal ideas, (irrelevant of how) to solve the specific brief. The students are therefore empowered in terms of their ability to control elements of the physical environment. Therefore educational outcomes of creativity, autonomy, fulfilment etc. are essential elements of a broad and balanced curriculum and illustrate how the Irish second Level system is striving to adopt skills and aptitudes that our rapidly changing global society necessitate.

\section{Integrating Design and Creativity}

The teaching of the generic skills of design and creativity draw on teaching methods that are of a modern philosophy and use varying pedagogies in order to stimulate learners to achieve in these areas. Peterson (2002) comments on how playfulness and humour in the classroom can contribute to the flow of ideas ${ }^{2}$. The intrinsic motivation required to promote creative thinking in pupils was increased through introducing pedagogical factors such as challenge and freedom ${ }^{3}$. However the nature of schooling can often hinder this type of approach. Guilford expressed the view that the development of creativity can be encouraged in schools but is inhibited by the conforming nature of schooling ${ }^{4}$. The mismatch between the rhetoric about the importance of conceptual aptitudes and the value placed on creative talent raises concern about the coherence of educational strategy. Education systems valuing educational managerial and administrative style skills over creativity in teaching ${ }^{5}$ highlights the misalignment between the direction and the objective of technological education. Sir Ken Robinson questioning if schools kill creativity states: "I believe our only hope for the future is to adopt a new conception of human ecology, one in which we start to reconstitute our conception of the richness of human capacity" 6 .

Even though the statements of intent of each technology subject is to promote the skills and attributes outlined in the previous section, the main driving motivation of the Irish second level pupil is success in a formal examination at the end of their course of study, with the emphasis on obtaining points for a national application system to access third level institutes. Admittedly, it is recognised that within all the senior cycle technology subject areas there is an element of the assessment that offers an opportunity for pupils to display their design and creative capabilities through practical coursework.

However these activities are advised to be carried out under pre-defined models/approaches of design with a number of mandatory steps. This leads pupils and teachers alike, to adopt a cautious approach that treats the stages and functions of design as hierarchical steps that the pupils are to be directly assessed on, creating a linear approach to an activity that should be treated much more globally ${ }^{7}$.

The paralysis of divergent thinking fuelled by assessment driven pedagogy and the anxiety surrounding the acquisition of credit to achieve third level entry, undermine the inclusion of design and creativity. Research at Harvard University found when 
studying high-functioning individuals that those who were "eminent creative achievers" demonstrated lower "latent inhibition" on standard psychological tests than average classmates ${ }^{8}$. Yet the structure of contemporary education mitigates against students taking creative risks and developing divergent thinking capacities.

\section{Resulting Educational Issues - Pedagogical and Learning Challenges}

Having considered the aims of contemporary technology education and the educational framework that we have inherited, the resulting challenges are inherently teaching and learning focused. The way in which technological teachers approach technology education and the responsibility that they carry as the main agents of change is of critical importance and one that is amplified in this paper as the cohort in question are pre-service technology teachers.

Firstly it is essential to recognise the importance of an individual acquiring adequate content knowledge in the subject area where creativity is to be achieved. The analogy of the subject matter forming building blocks that can be disassembled and reassembled as required to form a design idea, portrays the importance of comprehending subject specific content as a prerequisite. This pedagogical challenge is aided by ensuring that the learner has a clear context for the new material and can question their comprehension in terms of applied transferability.

\section{Method}

The research study employs a qualitative case study approach to explore a conventional modular approach to teaching process technologies and techniques. The study focused on two companion workshop-based modules that were traditionally rooted in the development of craft based skills (ie wood and metal working). Faced with the shift in focus from the acquisition of skills to the development of conceptual aptitudes, these modules necessitated a revision of teaching and learning activities. Broadening the educational experience and perspective of students within the constraints of traditional institutional structures demanded a clear aligning of educational needs with learning outcomes.

\section{Approach}

Understanding that design and creativity are "teachable" ${ }^{9}$ the research approach was modelled on the defined competencies essential to creative expression described by Robert Epstein ${ }^{10}$. This approach focused on providing students with the facility to capture inspiration, challenge their perception of technological education, develop divergent thinking skills, and explore and synthesis global stimuli.

The approach outlines three interrelated facets that ensure the facilitation of meaningful educational attainment.

1. Norms and Structures - Although it is possible to be creative outside your discipline or area of expertise, it is improbable. Deriving or establishing novel and meaningful links and relationships to form creative solutions is predicated by a fundamental knowledge and comprehension of the core building blocks within each discipline. The emphasis on design and creativity together with 
the development of craft based skills challenges the norms and practices of the conventional modular delivery. The approach taken in this study was to front load the development of the core skills in the initial half of the programme of work. The second half of the module then focused on students executing a semi-open design brief based on the initial skills developed. This structural shift was beneficial as:

a. The reorder of activities gives the students a context for the newly acquired knowledge and skills, as the semi-open brief was an exercise in 'near' and 'lateral' transferability.

b. Students were giving autonomy to design their workshop schedule, select appropriate process and materials to achieve their creative expression.

c. As the design challenge was presented at the beginning of the module students were given a scaffolded incubation period.

2. Communication and Expression - Moving from the traditional deliverables of a predefined, prescribed practical artefact, requires a defining of not only the narrative skills to communicate your creative inspiration and design but also the defining of what is deemed appropriate deliverables. With the emphasis on both the mastery of craft skills and creative expression it is important that the mode and medium used to respond to the task is appropriate. Students need the capacity to capture, manage, record and order information in an attempt to communicate the design journey. The approach taken was to utilize accessible personal technologies, i.e. their mobile phone. (ComReg's (Ireland's communication regulator) reported in December 2005 that mobile phone ownership reached $100 \%$ for the first time in September 2005) ${ }^{11}$. The benefits of integrating this technology to engineering design education are:

a. Students have the capacity to capture inspiration in real time. The creative activity that is core to the education objective of the design brief is no longer confined to or constrained by the scheduled environment or timetable of the module. The creative activity of designing is now an exercise in synthesising global inspiration.

b. The capacity and functionality of modern mobile phones enables students to capture information without judgment. Information can be later evaluated and synthesised. The integrated technology enhances the design process and acts as additional Pedagogist ${ }^{12}$.

c. The exploration of possible solution and the development of divergent thinking require a robust and flexible management system. Students need to capture, express and communicate information in the most appropriate manner for that information. The mode of representation will vary considerably across data sources, but more importantly needs to illustrate the idiosyncrasy of the students 'Presage, Process and Product' model of their learning process ${ }^{13} \& 14$.

d. Integrating a framework for teaching and learning that facilitates a comprehension of the learning style characteristics of engineering students enhances their application, motivation, level of engagement and quality of work ${ }^{15}$. Enabling students express their inspiration and design within a medium that is aligned with their preferential cognitive style enhances their capacity to push the boundaries of creative exploration. 
3. Values system - Expecting students to take a significant leap of faith and commit to a new philosophy of technological education without appropriate scaffolding, guidance and reassurance is improbable. Clearly defining the reciprocal relationship of 'design and make' empowers students to cognitively constructivist a synergy between both. The approach employs a semi-open design brief that requests students to design and make an A4 framed Scene, with the emotion of that scene expressed in a complementary flower artefact. The rationale for this design challenge was:

a. To seamlessly integrate the developed processing skills into a coherent set of artefacts. Thus enabling students transfer new knowledge and skill into a project that embodies their creative expression.

b. Female engagement in practical subjects in Ireland is significantly under represented (less that 5\% nationally). This male dominated bias reinforces a misconception of the holistic value of practical education. As the participant cohort in this study is student teachers it was important to challenge the perception of technological education and devise a non-traditional engineering brief.

c. The brief did not require students to discuss or present their designs under a series of predefined headings. Students were not given assessment criteria, but instead were encourage to establish what they perceived as important about their design solution and therefore were encourage to lead the assessor towards what they wanted assessed. In essence students were constructing their own value system in a concrete experiential activity.

\section{Participants}

The participant sample of 130 students represents $90 \%^{1}$ of the entire year one cohorts from the Materials and Engineering Technology and the Materials and Construction Technology initial teacher education degree programmes. Participants ranged in age from 17 to 32 with a mean age of 19.13 and a standard deviation of 2.97. Table 1 shows the homogeneity of both groups.

Table 1 - Homogeneity of the subgroups

\begin{tabular}{|cccccc|} 
& Course & Male & Female & $\begin{array}{c}\text { CAO } \\
\text { Entry* }\end{array}$ & $\begin{array}{c}\text { Mature } \\
\text { Entry }\end{array}$ \\
\hline M\&ET & $61(47 \%)$ & 61 & 0 & 54 & 7 \\
\hline M\&CT & $69(53 \%)$ & 68 & 1 & 66 & 3 \\
\hline $\begin{array}{l}\text { *CAO Entry - Refers to the standard entry route to third level education. } \\
\text { Office and are awarded entry based on performance in the Leaving Certificate Examinations (Performance calculated on the best } \\
\text { six grades achieved). }\end{array}$ \\
\hline
\end{tabular}

The participating cohort included $75.42 \%$ of students who had achieved their first choice college course. Although the remaining $24.58 \%$ of students were not studying their first choice programme, $70.83 \%$ of them had selected alternative teacher education courses as their first choice.

1 The remaining $10 \%$ were excluded from the study due to absenteeism during data collection sessions and natural attrition 


\section{Design and Implementation}

From the inception of the study to its completion, all facets were observed, recorded and reported by a team of researchers (including academics and teaching assistants). The design and implementation of this study addresses four sequential areas:

1. The types of mobile phone technology that the participant student body used and their ability to use that technology both as a capturing and communication tool.

2. The structure of the learning activities and the support procedures to ensure meaningful engagement.

3. Design of a conversion server that encourages creative expression by accommodating all file types and formats, while ensuring the uniformity of file format output as the building blocks for the students holistic design interface.

4. Utilise a holistic self-build web-based design interface that students can tailor to communicate their process of design and highlight their value system for assessment.

\section{Mobile phone functionality and operability audit}

At the beginning of the semester it was important to establish the value of integrating technology to enhance the creative activity and an audit was conducted to ascertain:

- The mobile phone functionality accessible to the participant group

- The participants normal phone usage

- The competency of the participant group to use both capturing and communication functions of their handset.

\section{Structure of learning activities}

The study was conducted over a 12 week semester and the completed task accounted for $50 \%$ of the academic credit for the modules.

The initial 6 weeks of the study focused on:

- Knowledge and skill development in the area of process technology, specifically decorative processing (defined by the module syllabi)

- Exploring the transferability of processes and tasks to establish the feasibility of achieving their desired creative expression.

- Explored stages and functions of design and analysed the design brief.

- Scaffolding of creative activities / synthesis / lateral thinking and inspiration

- Solving the design brief and planning the work schedule for the second part of the semester.

The second 6 weeks of the study focused on:

- Refining the creative direction of their project solution.

- Organising the material, processes, and requirements of their workshop activities.

- Executing their designed solution.

- Narrating their design journey, using an electronic diary collected throughout the process and building a unique holistic insight for assessment. 


\section{Conversion Infrastructure}

Students had access to a digital repository to facilitate the seamless transfer of data from their phones (or any other electronic device) to their own diary space. This infrastructure also supported all file types and the conversions of all file formats, thus enabling each student build their unique holistic interface. Figure 2 illustrates a schematic of the conversion infrastructure.

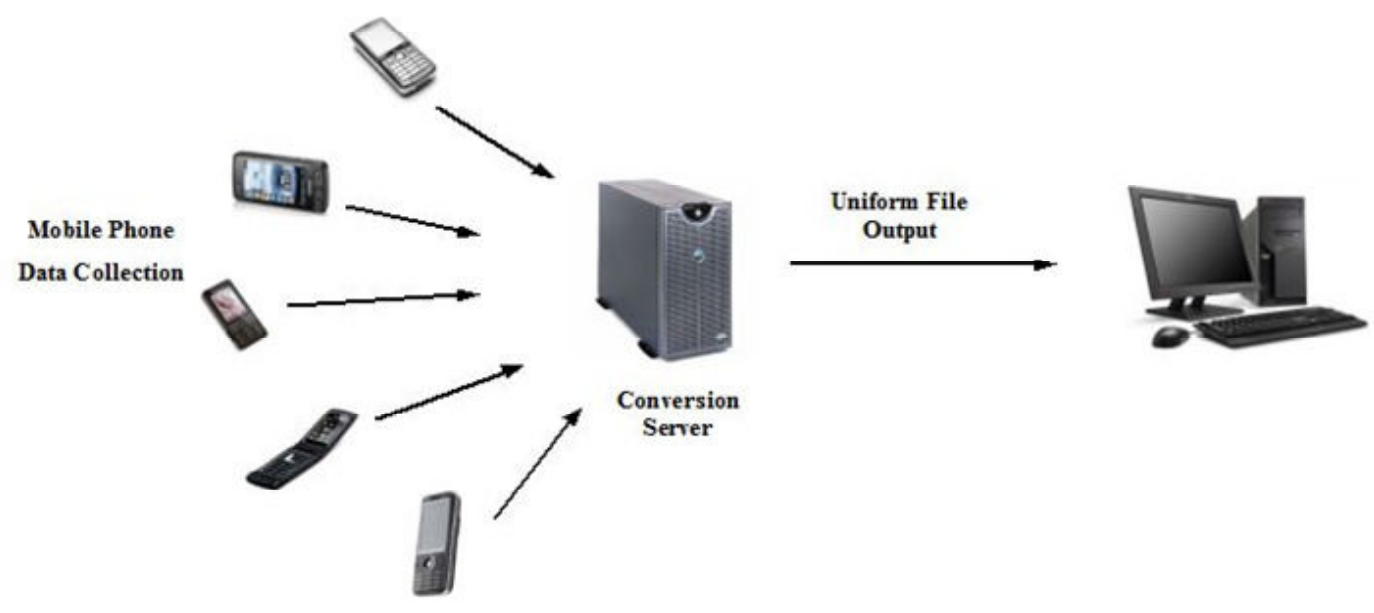

Figure 2 - Data conversion infrastructure

\section{Holistic assessment interface}

The difficulty with encouraging divergent thinking is the inability of traditional criterion referenced assessment to accurately measure the process of the activity. The measure beyond the artefact or finished product is critical to ensuring the sustainable value of concept driven competency.

A web based holistic interface together with the finished artefacts formed the assessment mechanism. This non-criterion reference formed the blank canvas for students to not only present the journey through designing the solution, but also leading the assessor towards what they valued about their solution(s).

- Students were given a blank web based interface with 24 blank panes and asked to populate the screen with the data required to explain their design process.

- Students only used the number of panes they deemed necessary to narrate their story (Figure $3)$.

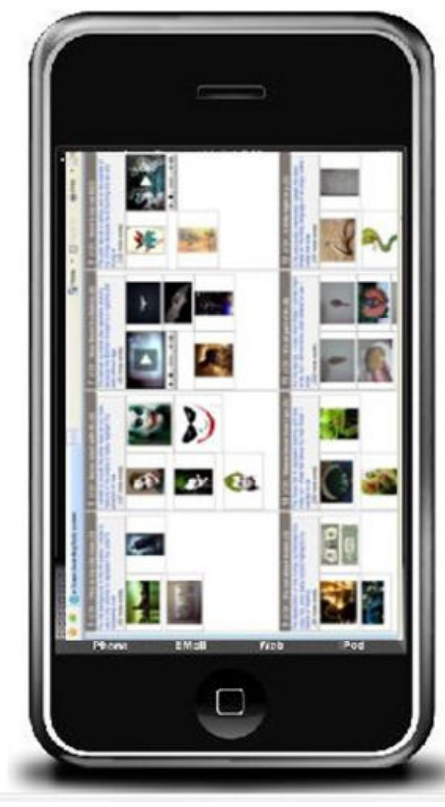

Figure 3 - Holistic design interface

- Data could be presented in any mode that they deemed appropriate. The site accommodated video, audio, image and text files. 
- The students in an attempt to illustrate the aspects that they perceived as important devised all headings on the individual panes and highlighted the areas that they believed identified the strength of their design solution or creative expression.

- Students could now express how they learned form their own mistakes, and critique their own design solution. Offering a new dimension to the assessment of the design activity.

- The ability to track the design process and gain an insight into the design inspiration and motivation, presents capabilities unattainable through traditional criterion referenced assessment.

\section{Results/Findings}

This section outlines the finding of the study.

\section{Results of Mobile phone functionality and operability audit}

All participants responded that they had a mobile phone with the majority of students owned a mobile phone for more than five years. See figure 4.

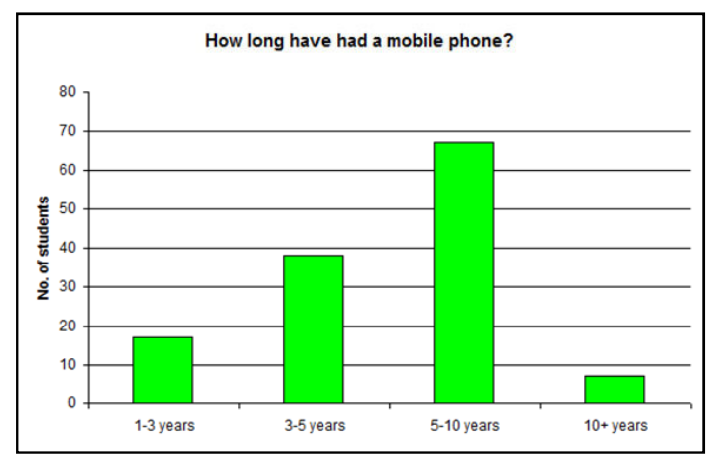

Figure 4 - Duration of mobile phone ownership

Students reported in figure 5 a strong capacity to send and receive image files. With just over $16 \%$ often or frequently use their phone to record graphical images.
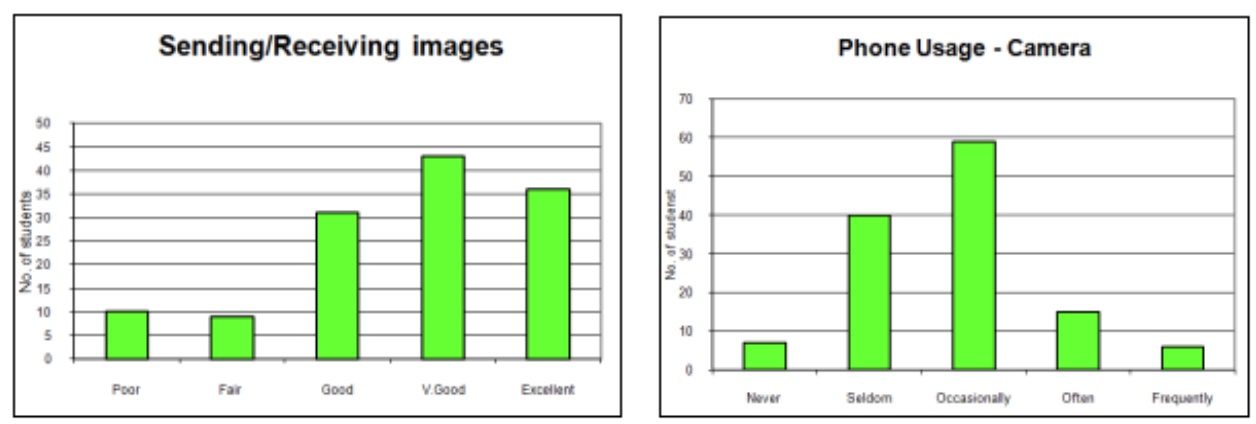

Figure 5 - Image functionality

The data collected to explore the capabilities and video functionality usage is similar to that of the camera usage. Although, students are aware of and can use the 
functionality the minority regularly use it, illustrated in figure 6.
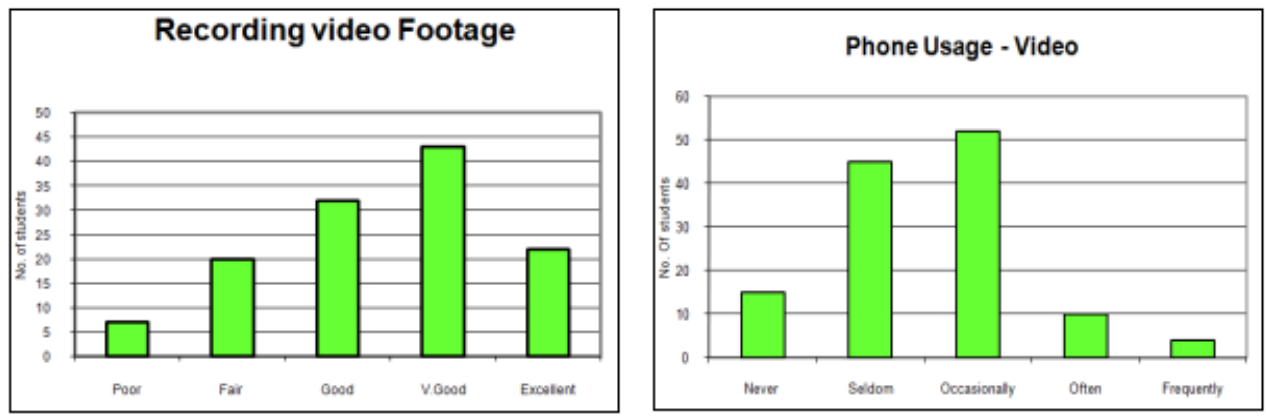

Figure 6 - Video functionality

Again there is no problem when using the phone as a data exchange device for audio files, figure 7 shows the lack of use of this functionality.
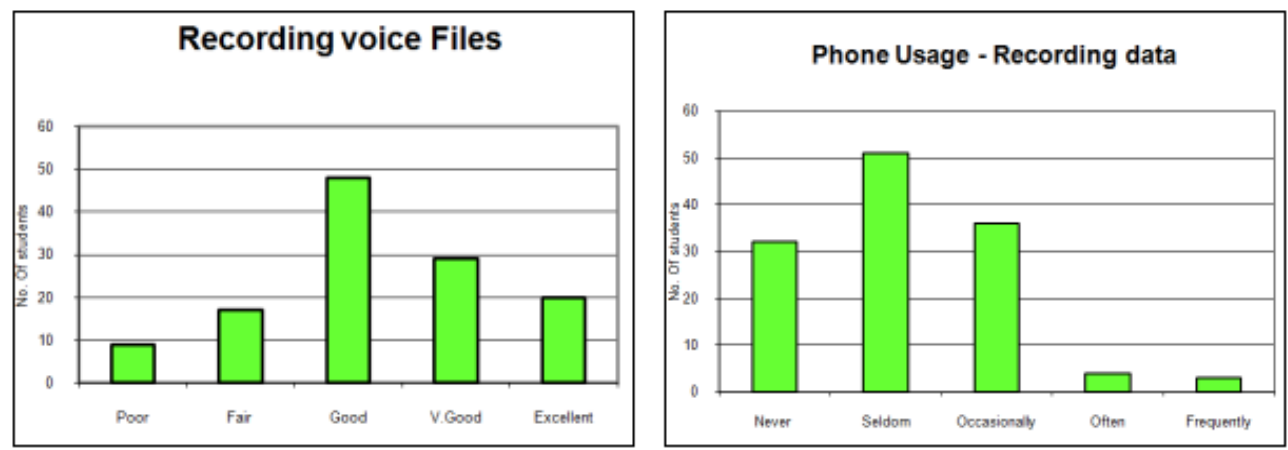

Figure 7 - Audio functionality

Contemporary mobile phones have significant capacity and the usage trends suggest the students are not using them to the full potential (Figure 8). Possibly the context or opportunity for meaningful usage has yet to be afforded to them.
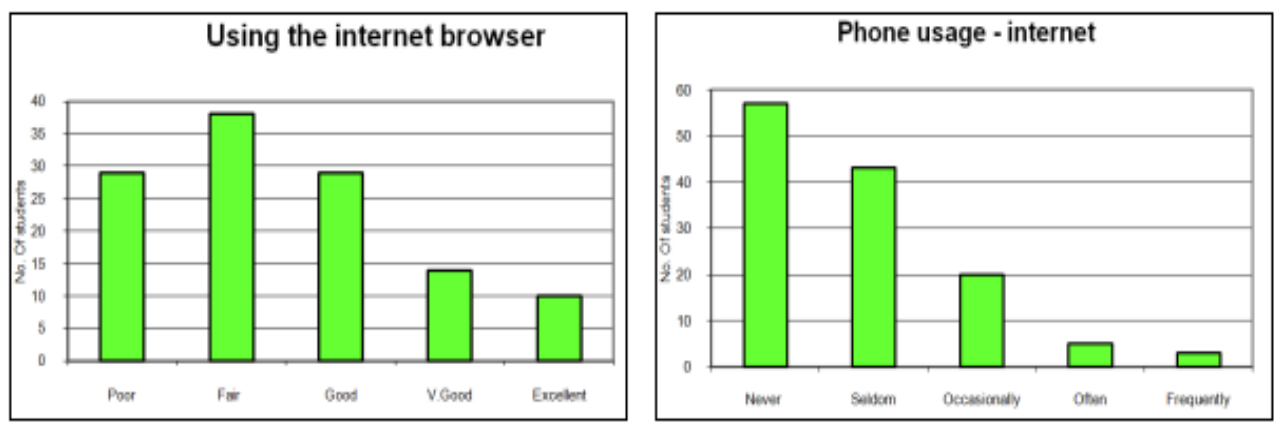

Figure 8 - Data conversion infrastructure 


\section{Levels of Engagement}

The module resulted in unprecedented lecture and laboratory attendance. The average lecture attendance was recorded at $88 \%$ (this is not accounting for students with genuine reasons for not attending). The high level of attendance resulted in a dynamic and enquiring lecture series that aided in exploration of the philosophical shift in technological education. For both the development phase and the self-directed phases of the workshop activities $100 \%$ attendance was recorded, as all students attended their scheduled laboratory sessions. However, over the self-directed phase of the study more the $25 \%$ of the cohort regularly attended additional laboratory session.

Over the course of the study each participating student produced on average 50 unique electronic files on their mobile phone and submitted them to their individual digital repository. Files were produced in real time, ranged form video to text and addressed all facets of their design activity (This is over twice the capacity of the holistic interface, even if they chose to use all available panes).

\section{Knowledge and skills}

Form the observations of the workshop activities it was noted that students were engaging in an additional activity by comparison to the traditional workshop experience. Students were making decisions, all students were deciding on materials that would best represent their design, processes were selected on the grounds of desired effect and students were prototyping. Experimentation with newly learned techniques created a constructivist comprehension of the core building blocks as students attempted to transfer knowledge and skills into their conceptual solution. The mastery of craft and processing skill was evident from all projects; students took ownership of the module and clearly benefited from the approach taken. Figure 9 demonstrates the mastery of engineering skills and the complexity of solutions.
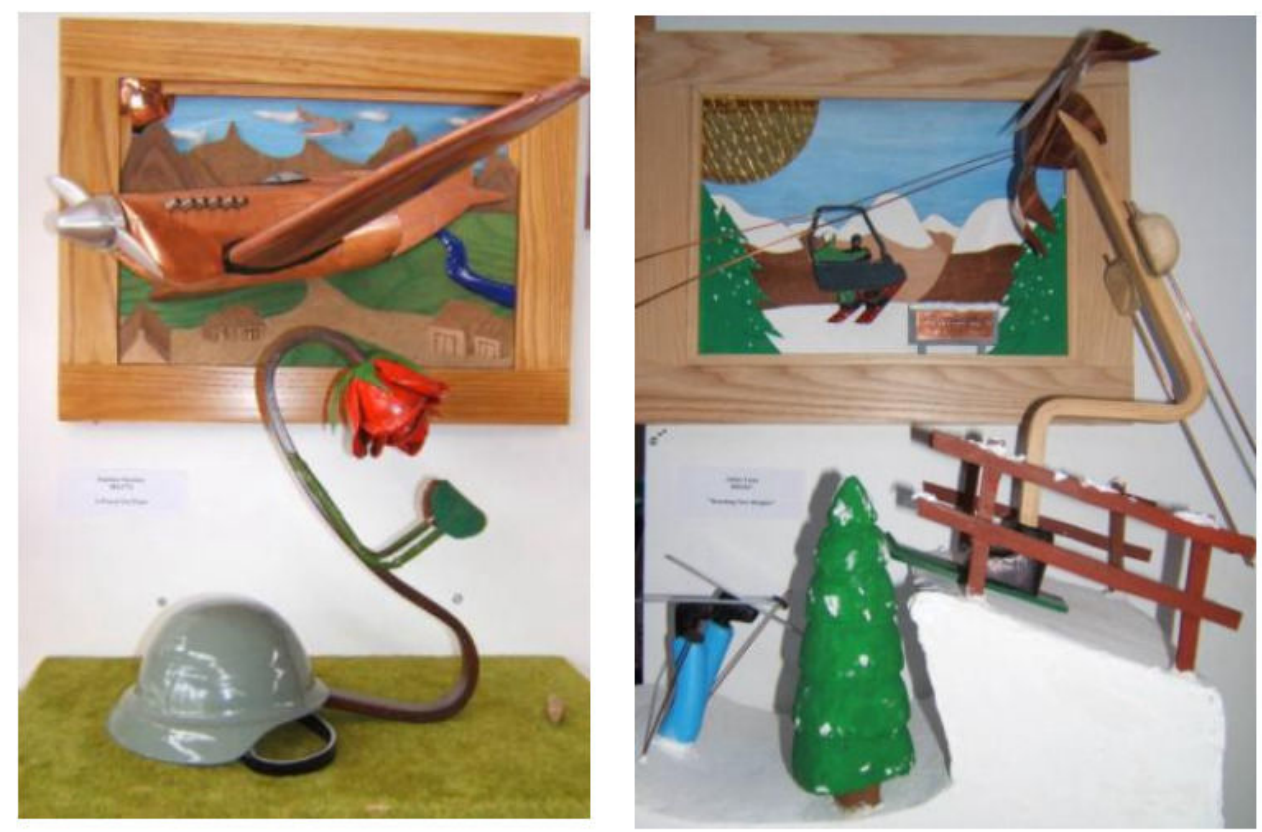

Figure 9 - Example of mastery of craft skills 
The solutions to the design brief clearly illustrated a deeper learning experience and the electronic portfolio validated this impression. The student in an attempt to tell their story specifically created the order of files illustrated in figure $10 \& 11$ these files are not in order of chorological creation.

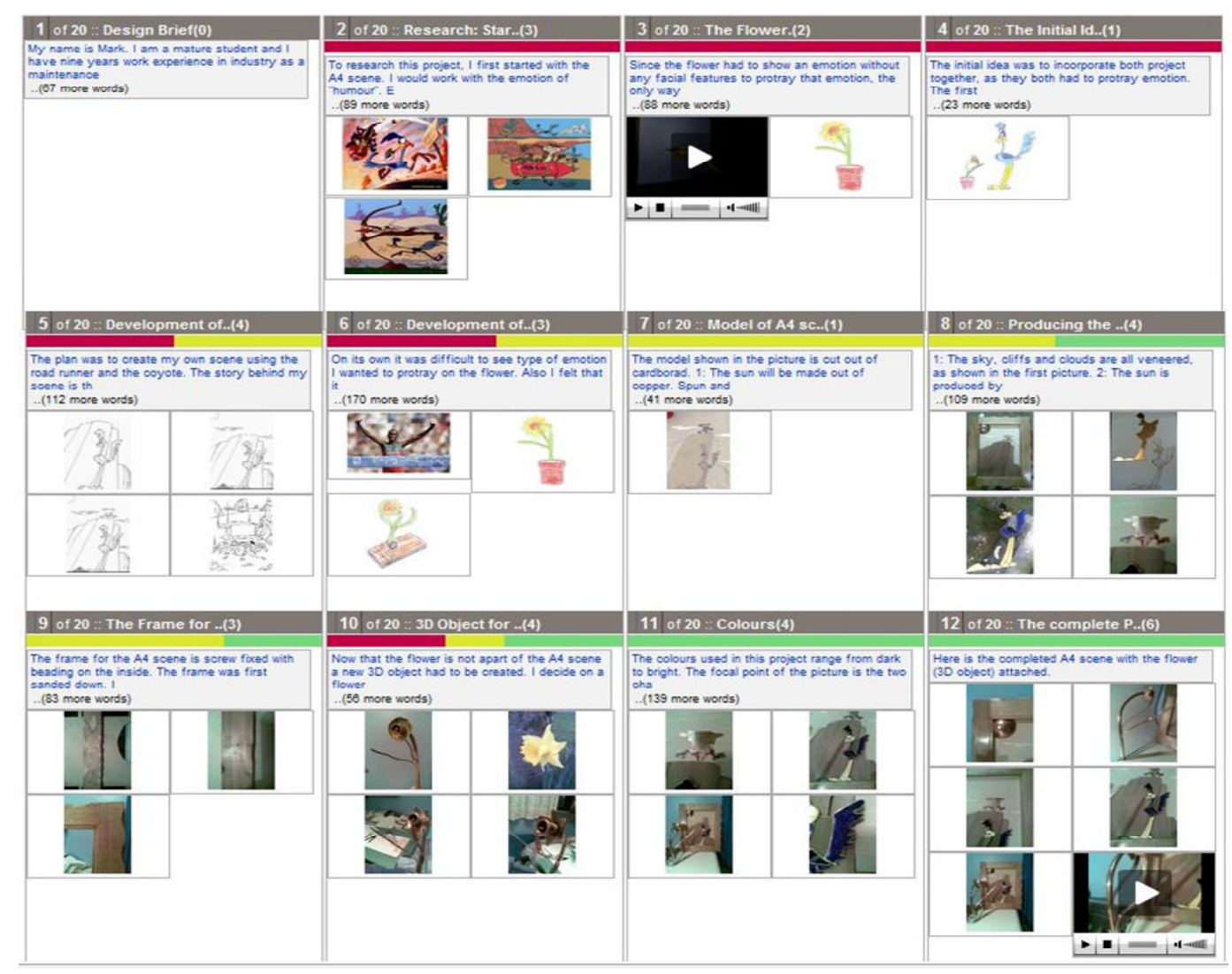

Figure 10 - Student defined holistic interface

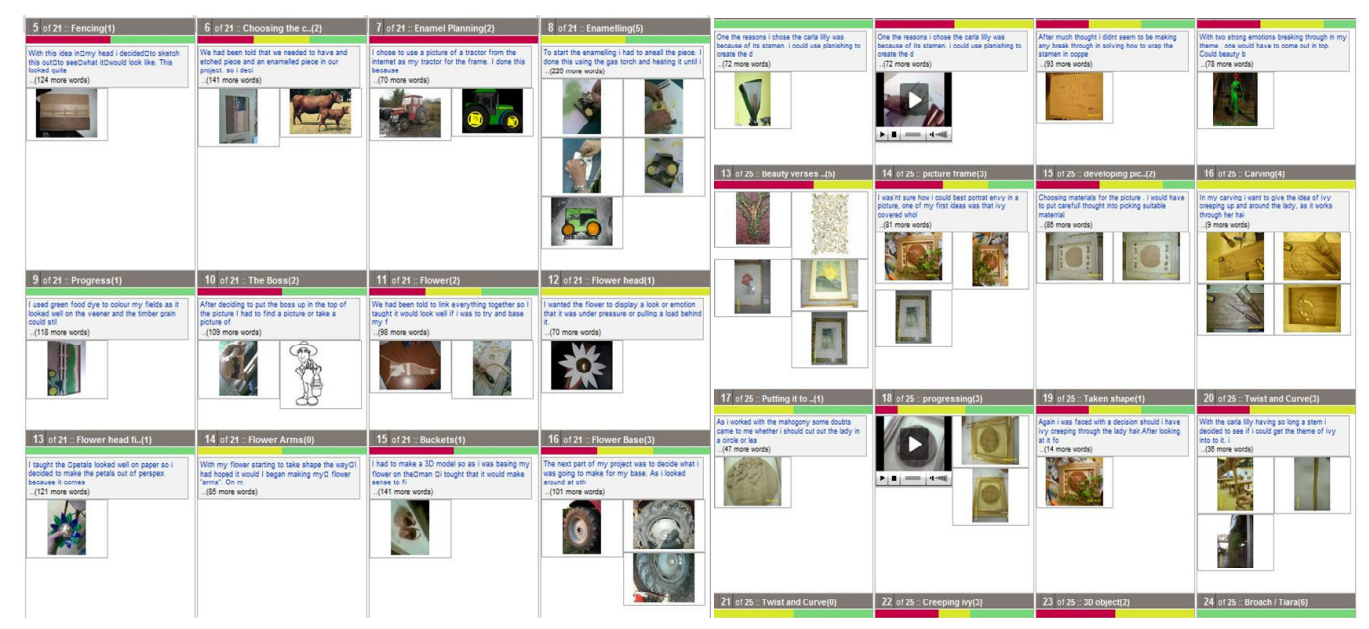

Figure 11 - Student defined holistic interface 


\section{Creative expression}

Each participating student produced an A4 picture frame with a scene designed and an accompanying flower that expressed the emotion of the scene. Students did not include facial expression to communicate the flowers emotion, instead utilised shape; form, colour, texture and material to achieve the desired emotional expression. The inclusion of emotion in the brief facilitated a personalised learning activity as the majority of the students utilised a personal experience. This served to capture a diverse range of emotions within the cohort and resulted in a powerful expression of the individually selected emotions, as there was an experiential comprehension. Figure 12 illustrates evidence of the diversity of emotion and the personalisation of project work.
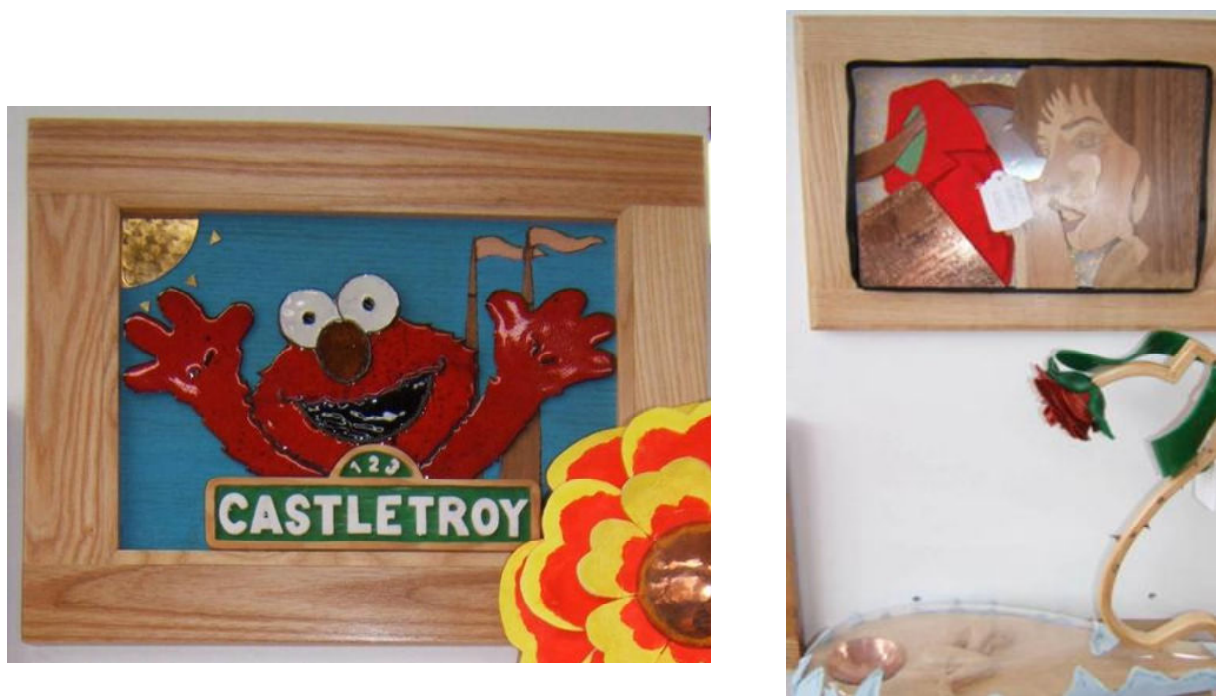

Figure 12 - Example of the emotional engagement

\section{$\underline{\text { Discussion }}$}

The dynamic nature of teaching and learning makes it difficult to capture and record the complexity of the design activity. The objective of the study was to inform teaching and learning in light of the demands of the new approach to technological education. This study explores the pedagogical implications of creative based activities and informs the contemporaneous role of the teacher /educator as they move from the instructor to the mentor.

The integration of technology as a significant element of the activity, enhanced, developed and empowered the student throughout the learning experience. However, there are also a number of areas that aided in maximising the quality of the design solutions and creative expression.

\section{Community of Learning}

Although ICT skills and the operability of the mobile phones were generally good, the findings illustrated that the majority of student teachers of technology were not utilizing the potential and functionality of their phones. This study presented students with the opportunity to utilise technology to support, manage and scaffold their learning. However on observation specific tasks challenged less experience 
participants, the response by the students was peer support. The workshops became a community of learning, as students positioned projects to be photographed, took video footage for each others work, shared blue tooth capabilities and supported each other in achieving their goals. A culture of teamwork and team building became evident within the workshop.

This evolution of collegiality among the class group is in contrast to prescribed traditionally focused activities. One hypothesis for the students' supportiveness is that no student felt as if they were trying to complete on predefined areas of the brief. As the activity was not determined by criterion reference, students were at liberty to share, critique and support the workshop based activities. Students understood that the value of the activity could not be effectively measured by a linear application of predefined criteria and therefore were not competing on that level.

\section{Valuing design education}

While the design solution validly measures the mastery of craft and process skills, and an observation of the creative expression combined with the emotion displayed by the flower artefact gives us an insight into creative capabilities, this is not the full story. The significant education values lies in the process of learning. The problems each student solved, the problems each student created and then solved, the inspiration for their expression, the synthesis of their ideas, and the emotional engagement that enabled students to personalise the experience, form the true value of design education. Structuring engineering design activities within structured educational paradigms is a challenge, but can be achieved if the learner is given a proactive part and not a spectator's role in their own learning.

\section{Autonomy of learning}

To support and sustain creativity within the technological subjects the teacher/educator must engage in innovative practice ${ }^{16}$. Including the learner in the creation of the learning experience plays a significant part in the affective engagement of that individual. The study illustrated how the students became the auditors and authors of there own learning experience. Learning developed from a paradigm of 'have to know' to 'need to know', as students were not only creating their own solutions but also their own problems. The essential transferability of new knowledge and skills fostered a deep engagement in the learning activity. The autonomy gained in the latter half of the module and the absence of criterion-referenced assessment reduced the anxiety of having to produce what is perceived to be required. Instead students could focus on defining and communication what they valued in a personalised learning activity.

Pink describes the 6 contemporary aptitudes required to thrive in the conceptual age ${ }^{17}$. Right brain dominant or conceptual aptitudes that become integral when the learning is personalised facilitate the ability of structured educational systems to develop aptitudes like Design, Story, Symphony, Empty, Play, and Meaning.

The value of creativity and design capabilities cannot be over stated when striving for a fast functioning knowledge economy. Although, it is possible to stimulate creative growth with the constraints of current educational structures, progress is needed to explore a fundamental rethink of our educational value system. 


\section{$\underline{\text { References }}$}

1. An Roinn Oideachas agus Eolaiochta, (2006) Technology Syllabus, Government Publication, Brunswick Press Ltd.

2. Peterson, R. E. (2002). Establishing the creative environment in technology education. Technology Teacher, 61(4), 7-10.

3. Amabile, T. M. (1998) How to Kill Creativity. Harvard Business Review 76, no. 5 (September-October 1998): 76-87.

4. Guilford, J. P. (1950). Creativity. The American Psychologist, 5(9), 444-454.

5. Kimbell, R. (2000). Creativity in Crisis. The Journal of Design and Technology Education, v5 (3) 206-211

6. Sir Ken Robinson says schools kill creativityTED2006, Filmed Feb 2006; Posted 2006 http://www.ted.com/talks/ken_robinson_says_schools_kill_creativity.html

7. Lewis, T. (2009). Creativity in technology education: providing children with glimpses of their inventive potential. International Journal of Technology Design Education 19 255-268.

8. Carson, S.H., Peterson, J.B, and Higgins, D.M. (2003) Decreased Latent Inhibition Is Associated With Increased Creative Achievement in High-Functioning Individuals. The Journal of Personality and Social Psychology, Vol. 85, No. 3, 499-506

9. Cameron, J. (2008) Let your creativity soar - Panel discussion. Scientific American Mind. 34

10. Epstein, R. (2000) The big book of creativity games. New York: McGraw-Hill,

11. ComReg (2005) Quarterly report forth quarter of 2005 (December)

12. Brookes, N. \& Hardy, A. (2002) The Craftplay Approach to Reggio Emilia. In Journal of Education in Museums. 2002 (23), pp. 20-23.

13. Biggs, J., (1993) What do inventories of students' learning processes really measure? A theoretical review and clarification. Br J Educ Psychol,. 63(Pt 1): p. 3-19.

14. Prosser, M. and K. Trigwell, (1999) Understanding Learning and Teaching: The Experience in Higher Education.

15. Felder and Brent (2005) Understanding Student differences Journal of Engineering Education 94(1), p.57-72

16. Wilson, V., Harris, M. (2004) Review of Effective Teaching and Learning of Design and Technology. International Journal of Technology and Design Education 223-241

17. Pink, D., (2005). A Whole New Mind: Why Right-Brainers Will Rule the Future the Berkely Publishing Group, Published by the Penguin Group. New York. 